\title{
Nouvelle politique éditoriale SFP-CNRS
}

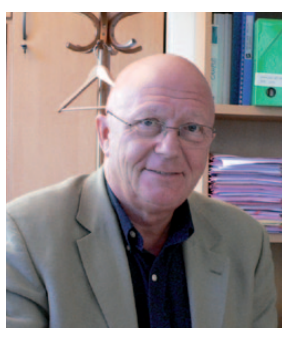

Michel Lannoo, Président de la Société Française de Physique

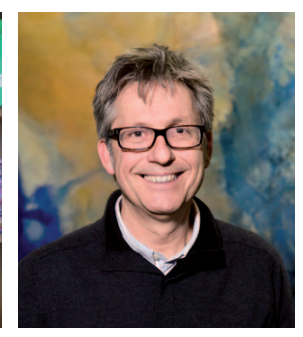

Jean-François Pinton, Directeur de l'Institut de Physique du CNRS

La Société Française de Physique (SFP) et I'Institut de Physique du CNRS (INP) mènent actuellement plusieurs actions en commun visant à rassembler plus largement la communauté des physiciens. Il s'agit de promouvoir notre discipline auprès d'un public aussi large que possible, afin d'en faire saisir tous les enjeux et d'attirer davantage de jeunes. Les physiciens sont très présents dans les progrès pluridisciplinaires et la physique est, et a toujours été, à l'origine de nombreuses innovations aux conséquences sociétales importantes.

La revue que vous tenez entre les mains est le fruit de la volonté de mener une politique éditoriale commune, en rapprochant Reflets de la physique (SFP) et Images de la physique (CNRS). Sous des formats différents, ces deux revues étaient destinées au même lectorat et attiraient les mêmes contributeurs potentiels. Nous avons fait le choix de mettre en commun nos moyens pour la rédaction, la réalisation et la promotion de ces deux revues afin de produire un seul média plus riche en contenu et mieux diffusé. Les articles de fond qui ont marqué les Images viennent enrichir le contenu traditionnel des Reflets. La collaboration SFP/CNRS, à travers les instituts couvrant les domaines de la physique (INSIS, IN2P3, INSU et INP), doit permettre l'émergence de nombreux sujets - avec un large spectre des thèmes traités. L'année 2013 sera consacrée à mettre en place la nouvelle ligne éditoriale, avec un comité de rédaction unique.

Dans ce premier numéro commun, vous découvrirez notamment deux articles longs sur le thème des rayons cosmiques, dont le centenaire de la découverte a été célébré en 2012, un article dans la série en cours sur la supraconductivité, un article sur le dernier prix Nobel de physique, le point sur ITER et deux articles d'histoire des sciences. Vous lirez également les rubriques habituelles de Reflets de la physique (nouvelles de la SFP, note de lecture, etc.), le portrait d'une physicienne du laboratoire de physique des solides d'Orsay ou encore un retour sur les Journées des Entrants dans les laboratoires de l'institut de PHYsique, initiative conjointe de I'INP, de la SFP et de la SFO. Le numéro publiera aussi une prise de position de la SFP et de l'INP sur la « Sensibilisation des physiciens à l'utilisation abusive des facteurs d'impact».

Avec 5 numéros par an, la nouvelle revue sera diffusée sur abonnement, aux meilleures conditions pour les adhérent-e-s de la SFP, et au tarif préférentiel de $37 €$ pour les personnels des unités de recherche CNRS.

Ce premier numéro est diffusé gratuitement à l'ensemble des destinataires des revues Reflets de la physique et Images de la physique, pour faire connaître la nouvelle formule. Nous invitons nos lecteurs à manifester leur intérêt en remplissant et en retournant à la SFP l'encart contenu dans ce numéro ou en s'abonnant sur le site www.refletsdelaphysique.fr. Ce point est essentiel : faire vivre une revue de qualité, au service de la communauté et de la diffusion de ses avancées, ne peut se faire sans la participation des acteurs concernés.

Nous voulons parier que l'attractivité de la nouvelle formule de la revue s'accompagnera également $d^{\prime}$ une forte augmentation des adhésions à la SFP. Ce rapprochement s'inscrit en effet dans une volonté d'élargir et de mieux souder la communauté des physiciens. Dans les échanges avec les partenaires institutionnels, dans la promotion de la recherche en physique, il est impératif de nous rassembler et d'agir.
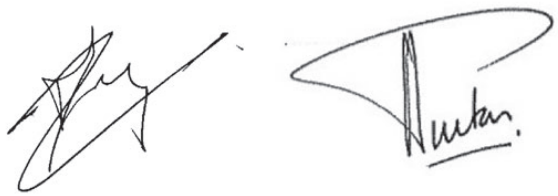